\title{
NDV-D90 suppresses growth of gastric cancer and cancer-related vascularization
}

\author{
Hong Sui ${ }^{1, *}$, Kaibing Wang ${ }^{2, *}$, Rui Xie ${ }^{1, *}, \mathbf{X i} \mathbf{L i}^{3}$, Kunpeng $\mathrm{Li}^{3}$, Yuxian Bai ${ }^{1}$, Xishan \\ Wang ${ }^{4}$, Bin Bai ${ }^{2}$, Dan Chen ${ }^{1}$, Jiazhuang $\mathrm{Li}^{1}$, Baozhong Shen ${ }^{5}$ \\ ${ }^{1}$ Department of Medical Oncology, Harbin Medical University Cancer Hospital, Harbin 150040, China \\ ${ }^{2}$ Department of Intervention, The Second Hospital Affiliated Harbin Medical University, Harbin 150086, China \\ ${ }^{3}$ Division of Swine Disease, National Key Laboratory of Veterinary Biotechnology, Harbin Veterinary Medicine, Harbin 150069, China \\ ${ }^{4}$ Department of Abdominal Surgery, Cancer Hospital, Chinese Academy of Medical Sciences and Peking Union Medical \\ College, Beijing 100021, China \\ ${ }^{5}$ Department of Radiology, The Fourth Hospital of Harbin Medical University, Harbin 150001, China \\ *These authors contributed equally to this work and are joint First authors \\ Correspondence to: Baozhong Shen, email: shenbaozhong2016@163.com \\ Hong Sui, email: suihong2003@yeah.net
}

Keywords: gastric cancer, newcastle disease viruses (NDV), apoptosis, vascular endothelial growth factor A (VEGF-A), matrix metallopeptidase 2 (MMP-2)

Received: March 01, 2017

Accepted: March 15, 2017

Published: March 25, 2017

Copyright: Sui et al. This is an open-access article distributed under the terms of the Creative Commons Attribution License (CC-BY), which permits unrestricted use, distribution, and reproduction in any medium, provided the original author and source are credited.

\section{ABSTRACT}

Recent reports suggest promises on using oncolytic Newcastle disease viruses (NDV) to treat different cancers, while the effects of a NDV-D90 strain on gastric cancer remain unknown. Here we showed that NDV-D90 induced gastric cancer cell apoptosis in a dose-dependent manner in 3 gastric cancer cell lines BGC-823, SGC-7901 and MKN-28. Pronounced reduction in cell invasion was detected in NDVD90-treated BGC-823 and SGC-7901 cells, but not in MKN-28 cells. The increases in cell apoptosis and reduction in cell growth in NDV-D90-treated gastric cancer cells seemingly resulted from augmentation of p38 signaling and suppression of ERK1/2 and Akt signaling. In vivo, orthotopic injection of NDV-D90 impaired tumor growth and induced intratumoral necrosis. Tumor cells that had been pre-treated with NDV-D90 showed defect in development of implanted tumor. Moreover, NDV-D90 appeared to reduce gastric tumor vascularization, possibly through suppression of vascular endothelial growth factor A and Matrix Metallopeptidase 2. Together, our data suggest that NDV-D90 may have potential anti-cancer effects on gastric cancer.

\section{INTRODUCTION}

Gastric cancer is a prevalent cancer, in which malignant cells form in the lining of the stomach [1]. Nearly all gastric cancers are adenocarcinomas [2]. Although surgical removal of the part of stomach that bares tumor may be effective for early stage gastric cancer, the majority of gastric cancer patients are diagnosed at an advanced stage due to lack of early signs or symptoms of the disease [1]. Therefore, novel therapies are required for treatment of advanced gastric cancer that may be amendable by surgery.

The anti-cancer potential of Newcastle disease viruses (NDV) has been discovered since 1805s [3].
The selective targeting elimination of replicating tumor cells by NDV stem from the presence of defective Interferon signaling pathways in tumor cells, but an effective antiviral response to hamper viral replication in normal cells [3]. Recently, selection, modification and production of oncolytic NDV with minimal damage of the normal adjacent tissues have been achieved [4, 5]. Genetic editing of NDV to express immune modulators (i.e. GM-CSF) has amplified the effects of NDV on targeted cancer tissues, rendering them one of the most effective multitarget cancer vaccines [5]. Since the clinical study of oncolytic NDV, three strains of NDV, MTH-68 [6], NDVHUJ [7] and PV701 [8-11] have now been used in phase I/ II clinical trials for tumor treatment. 
Very recently, a NDV-D90 strain that was isolated from natural sources in China, and was shown to specifically induce apoptosis of human lung adenocarcinoma cell line A549 through regulating caspases [12]. Moreover, application of NDV-D90 appeared to reduce tumor growth in vivo [13]. Furthermore, the anti-cancer effects of NDV-D90 were just reported in oral squamous cell carcinoma [14]. However, whether NDV-D90 may have similar effects on gastric cancer is unknown.

Here, we addressed this question. We found that NDV-D90 induced gastric cancer cell apoptosis in a dosedependent manner in 3 gastric cancer cell lines BGC-823, SGC-7901 and MKN-28. Pronounced reduction in cell invasion was detected in NDV-D90-treated BGC-823 and SGC-7901 cells, but not in MKN-28 cells. The increases in cell apoptosis and reduction in cell growth in NDVD90-treated gastric cancer cells seemingly resulted from augmentation of $\mathrm{p} 38$ signaling and suppression of ERK1/2 and Akt signaling. In vivo, orthotopic injection of NDV-D90 impaired tumor growth and induced intratumoral necrosis. Tumor cells that had been pretreated with NDV-D90 showed defect in development of implanted tumor. Moreover, NDV-D90 appeared to reduce gastric tumor vascularization, possibly through suppression of vascular endothelial growth factor A (VEGF-A) and Matrix Metallopeptidase 2 (MMP-2). Together, our data suggest that NDV-D90 may have potential anti-cancer effects on gastric cancer.
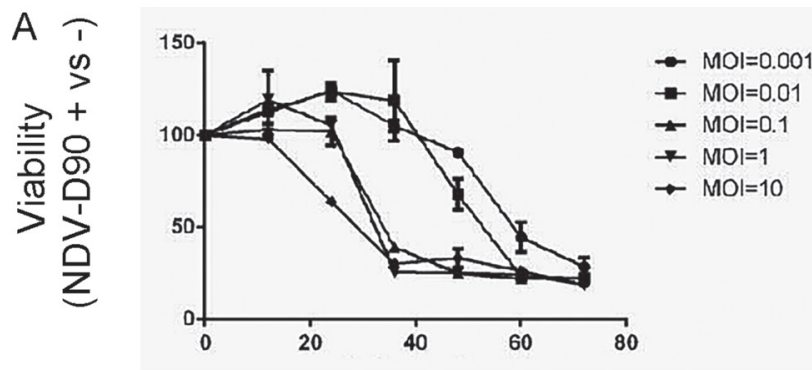

BGC-823 post-infection time (h)

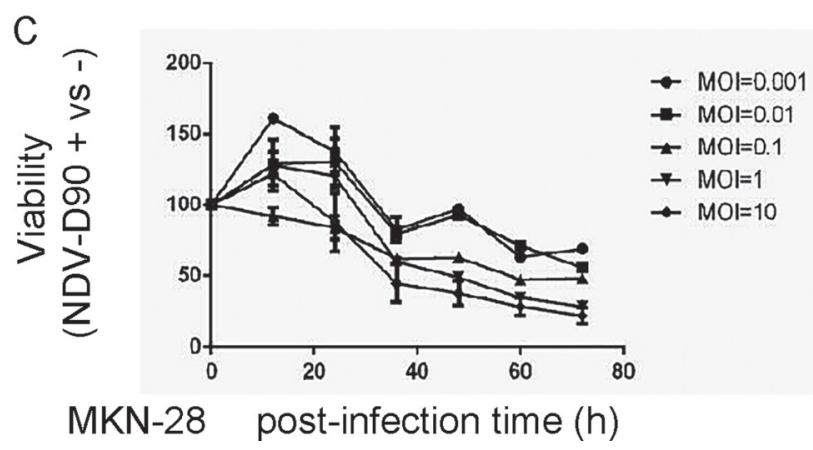

\section{RESULTS}

\section{NDV-D90 reduces gastric cancer cell growth in vitro}

First, we examined the effects of NDV-D90 on the growth of gastric cancer cells in vitro. We used 3 human gastric cancer cell lines, BGC-823, SGC-7901 and MKN-28. BGC-823 is low differentiated gastric carcinoma, SGC7901 is medium differentiated gastric carcinoma, while MKN-28 is highly differentiated gastric carcinoma. We found that NDV-D90 dose-dependently reduced the cell viability in all 3 lines, and the effects of NDV-D90 on BGC823 and SGC-7901 cells appeared to be more pronounced (Figure 1A-1C). Next, virus amplification was measured in the 3 cell lines, showing that viruses proliferated more quickly in BGC-823 and SGC-7901 cells, compared to MKN-28 cells (Figure 1D). Together, these data suggest that NDV-D90 reduces gastric cancer cell growth in vitro, and NDV-D90 may be more effective on low differentiated, highly proliferative gastric cancer cells.

\section{NDV-D90 reduces gastric cancer cell invasion in vitro}

We then examined the alteration of gastric cancer cell invasion after NDV-D90 treatment. Tumor cell invasion test was performed and the invasion index (here
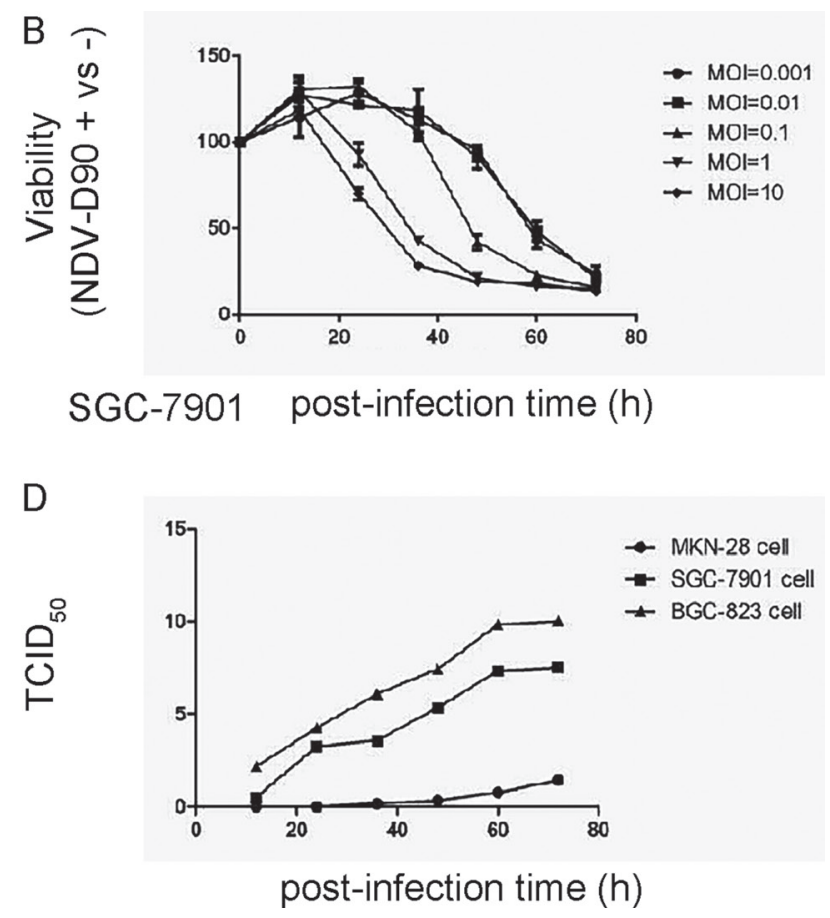

Figure 1: NDV-D90 reduces gastric cancer cell growth in vitro. We treated 3 gastric cancer cell lines, BGC-823, SGC-7901 and MKN-28, with different MOI of NDV-D90, and analyzed cell viability at different time points in an CCK-8 assay. (A) CCK-8 assay for BGC-823. (B) CCK-8 assay for SGC-7901. (C) CCK-8 assay for MKN-28. (D) TCID ${ }_{50}$ for virus amplification in 3 cell lines. The amount of a pathogenic agent that will produce pathological change in $50 \%$ of cell cultures inoculated is expressed as $\mathrm{TCID}_{50} / \mathrm{ml}^{*}{ }^{*}<<0.05$ : BGC-823 vs MKN-28. * $p$ < 0.05: SGC-7901 vs MKN-28. $N=5$. 
termed as the ratio of the NDV-D90-treated tumor cells that pass through the chamber versus the control tumor cells that pass through the chamber) was determined. We found that NDV-D90 treatment reduced the invasion index by $83 \%$ in BGC- 823 cells, by $85 \%$ in SGC-7901 cells, but only by $20 \%$ in MKN-28 cells, shown by representative images (Figure 2A), and by quantification (Figure 2B). These data suggest that NDV-D90 reduces gastric cancer cell invasion in vitro and this effect may be more pronounced on low differentiated, highly proliferative gastric cancer cells.

\section{NDV-D90 induces gastric cancer cell apoptosis in vitro}

Next, we analyzed the effects of NDV-D90 on cell apoptosis. We found that NDV-D90 induced apoptosis in a dose-dependent manner in all 3 gastric cancer cell lines BGC-823 (Figure 3A-3B), SGC-7901 (Figure 3C-3D) and MKN-28 (Figure 3E-3F). Again, more pronounced reduction in cell invasion was detected in NDV-D90treated BGC-823 and SGC-7901 cells, but not in MKN-28 cells (Figure 3A-3F).

\section{NDV-D90 enhances p38 signaling and inhibits ERK1/2 and Akt signaling in gastric cancer cells}

In order to understand the underlying mechanisms, we analyzed phosphorylation of a key factor in apoptosis- associated signaling pathway, p38, phosphorylation of 2 key factors in proliferation-associated signaling pathway, ERK1/2 and AKT. We found that NDV-D90 treatment induced the phosphorylation of $\mathrm{p} 38$, but suppressed the phosphorylation of ERK1/2 and AKT on BGC-823 cells (Figure 4). These data suggest that the increases in cell apoptosis and reduction in cell growth in NDV-D90-treated gastric cancer cells may result from augmentation of p38 signaling and suppression of ERK1/2 and Akt signaling.

\section{NDV-D90 reduces gastric cancer cell growth in vivo}

The next question is whether NDV-D90 may have similar anti-cancer effects against gastric cancer in vivo. In order to examine the implanted tumor cells in living animals, we transduced

BGC-823 cells with lentivirus carrying a RFP reporter (Figure 5A-5D). The transduced cells (termed as BGC-823-RFP) were validated under fluorescent microscopy in vitro (Figure 5E). BGC-823-RFP cells were then implanted into nude mice, after which NDV-D90 was intratumorally injected. The tumor was monitored at different time points after viral injection, showing suppression of tumor growth by representative images (Figure 5F). Significant necrosis was detected exclusively in the implanted BGC-823-RFP tumor treated with NDV-D90 (Figure 5G). When BGC-823-RFP cells were pre-treated with NDV-D90 before implantation, we found
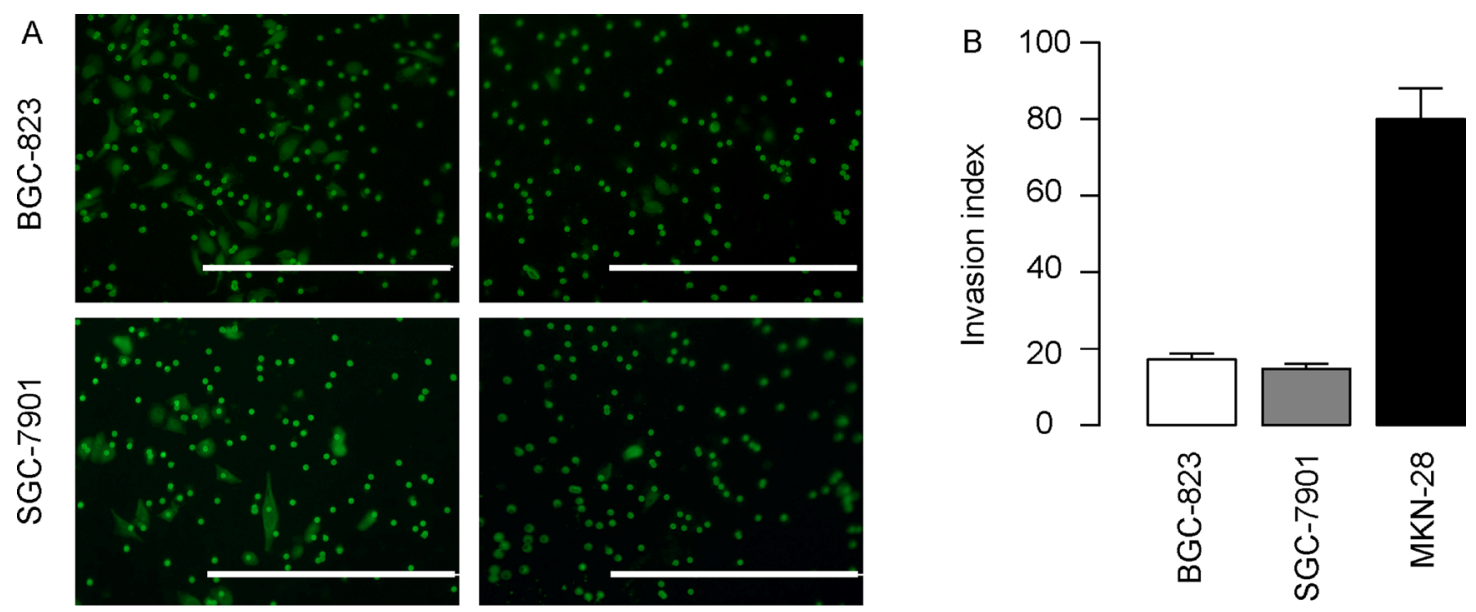

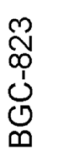

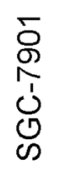

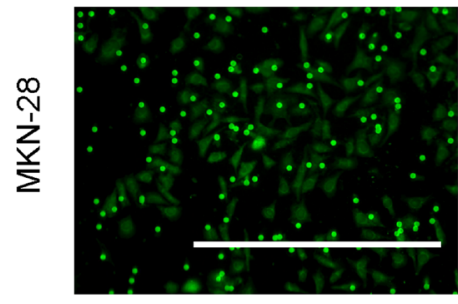

- NDV-D90

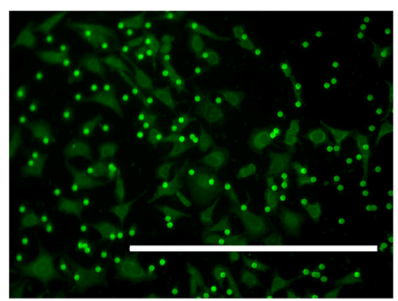

+ NDV-D90

Figure 2: NDV-D90 reduces gastric cancer cell invasion in vitro. Tumor cell invasion test was performed and the invasion index (here termed as the ratio of the NDV-D90-treated tumor cells that pass through the chamber versus the control tumor cells that pass through the chamber) was determined. (A) Representative images. (B) Quantification. Scale bars are $200 \mu \mathrm{m} . N=5$. 
that 48 hours after transplantation, the signals from the implanted tumor cells were hardly detected (Figure $5 \mathrm{H}$ ). Together, these data support an anti-cancer role of NDV-D90 in vivo in gastric cancer.

\section{NDV-D90 impairs gastric cancer vascularization}

Finally, we examined the effects of NDV-D90 on gastric cancer vascularization. Implanted tumors treated with/without NDV-D90 were dissociated and analyzed for the percentage of CD31+ endothelial cells inside the tumor. We found that NDV-D90 treatment significantly reduced the percentage of the CD31+ endothelial cells inside the tumor, shown by representative flow charts (Figure 6A), and by quantification (Figure 6B). We then examined the regulators of vascularization in the tumor and found that the levels of VEGF-A were significantly reduced in NDV-D90-treated tumor, compared to control,
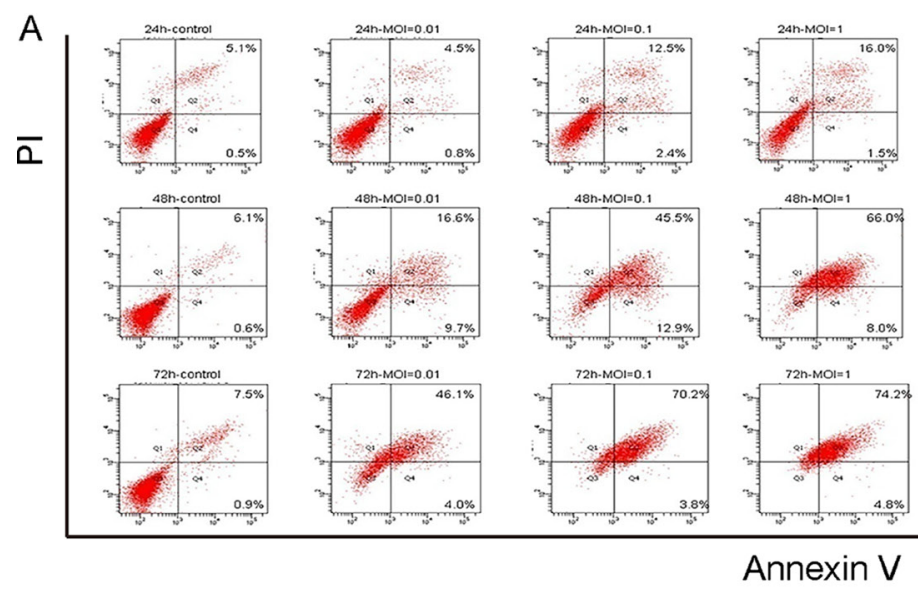

$C$
$\bar{n}$
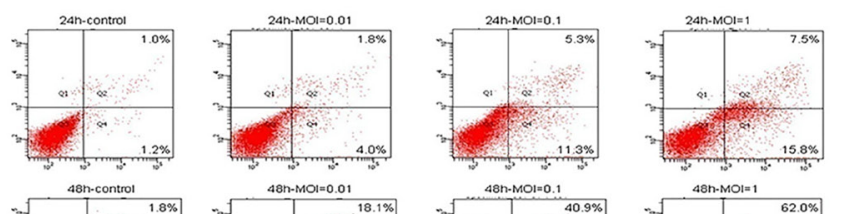

$\bar{\alpha}$
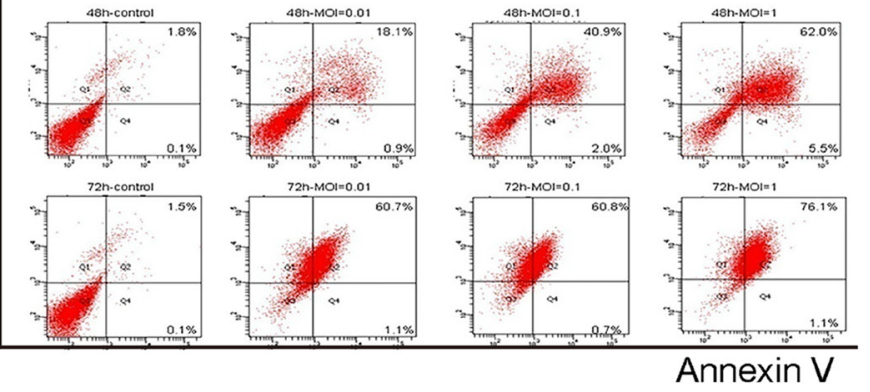

$E$
$\bar{a}$
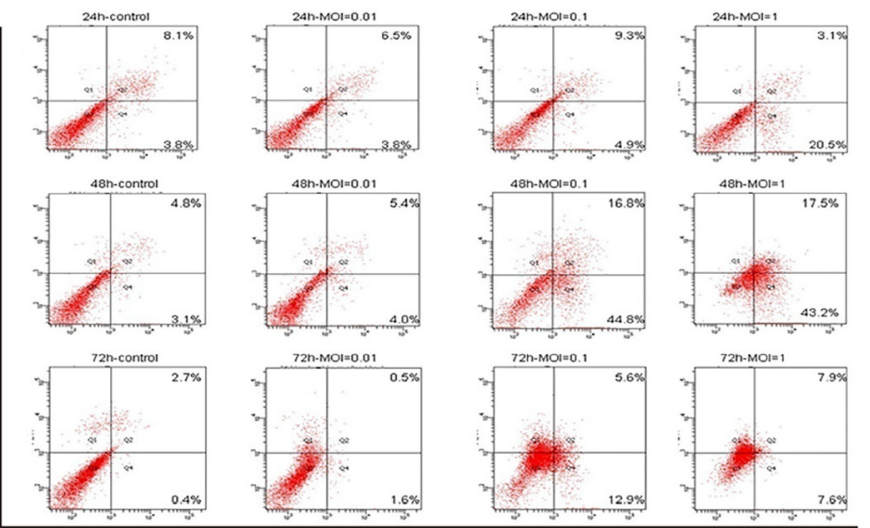

Annexin V
B

BGC-823

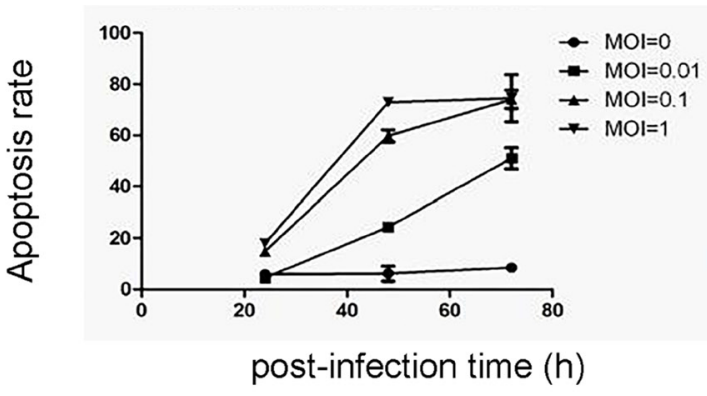

D

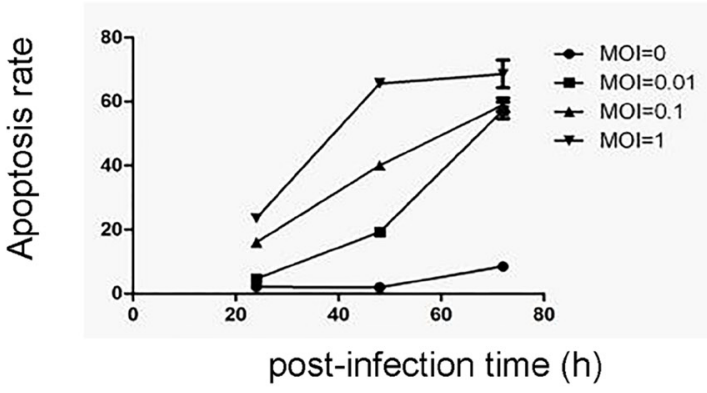

$\mathrm{F}$

MKN-28

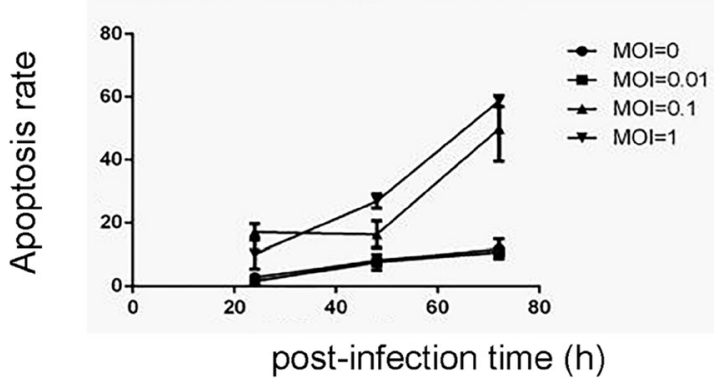

Figure 3: NDV-D90 induces gastric cancer cell apoptosis in vitro. The effects of NDV-D90 on cell apoptosis were analyzed by Annexin V / PI assay using flow cytometry. (A) Representative flow charts for BGC-823. (B) Quantification for BGC-823. (C) Representative flow charts for SGC-7901. (D) Quantification for SGC-7901. (E) Representative flow charts for MKN-28. (F) Quantification for MKN-28. $N=5$. 
by ELISA (Figure 6C) and by immunohistochemistry (Figure 6D). Similarly, we found that the levels of MMP-2 were significantly reduced in NDV-D90-treated tumor, compared to control, by ELISA (Figure 6E) and by immunohistochemistry (Figure 6F). Thus, NDV-D90 appeared to reduce gastric tumor vascularization, possibly through suppression of VEGF-A and MMP-2. Together, our data suggest that NDV-D90 may have potential anticancer effects on gastric cancer.

\section{DISCUSSION}

NDV is also known as avian paramyxovirus serotype 1, which belongs to a non-segmented, negativestrand RNA virus of the family Paramyxoviridae with a natural avian host range [3]. The genome of NDV is approximately $15 \mathrm{~kb}$ in length that encodes 6 structural proteins. Recent advance in application of virulence associated with human pathogens renders animal viruses as a promising therapeutic agent for tumor, since NDV does not have significant adverse effects in humans [3]. Interestingly, the selective targeting and the destruction of replicative tumor cells by NDV seemingly originates from the defective interferon signaling pathways in tumor cells, since interferon regulatory factor genes encode DNAbinding proteins that are involved in the innate immune response to viral infection [15].

Very recently, NDV-D90 strain was shown to induce apoptosis of human lung cancer cells and inhibit tumor growth in vitro [12], and in vivo [13]. In another independent study, the anti-cancer effects of NDV-D90 were detected in oral squamous cell carcinoma. However, whether NDV-D90 may have similar effects on gastric cancer is unknown. Moreover, the underlying mechanisms remain ill-defined. Here, we addressed these questions.

We found that NDV-D90 induced gastric cancer cell apoptosis and reduced cell invasion in a dose-dependent manner in 3 gastric cancer cell lines BGC-823, SGC-7901 and MKN-28. However, pronounced effects were detected in NDV-D90-treated BGC-823 and SGC-7901 cells, but not in MKN-28 cells. Since BGC-823, SGC-7901 and MKN-28 represent low differentiated, medium differentiated and highly differentiated gastric cancer cells respectively, these data suggest that NDV-D90 may be more effective on low differentiated, highly proliferative gastric cancer cells, which is supported by the high amplification of NDV-D90 in low differentiated, highly proliferative gastric cancer cells.

We detected suppression of VEGF-A and MMP-2 in NDV-D90-treated gastric cancer cells. Both VEGF-A and MMP-2 are associated with tumor-related vascularization [16-19]. Of note, MMP-2 was regulated by p38, ERK1/2 and Akt signaling pathway [20-22], while VEGF-A was similarly regulated by p38, ERK1/2 and Akt signaling pathway [23-25]. Therefore, although the increases in cell apoptosis and reduction in cell growth in NDVD90-treated gastric cancer cells seemingly resulted from augmentation of apoptosis-associated p38 signaling and suppression of proliferation-associated ERK1/2 and Akt signaling, these signaling pathways also have effects on cell mobility and invasion potential.

Finally, we approved our in vitro findings in vivo. Orthotopic injection of NDV-D90 impaired tumor growth and induced intratumoral necrosis. The effects

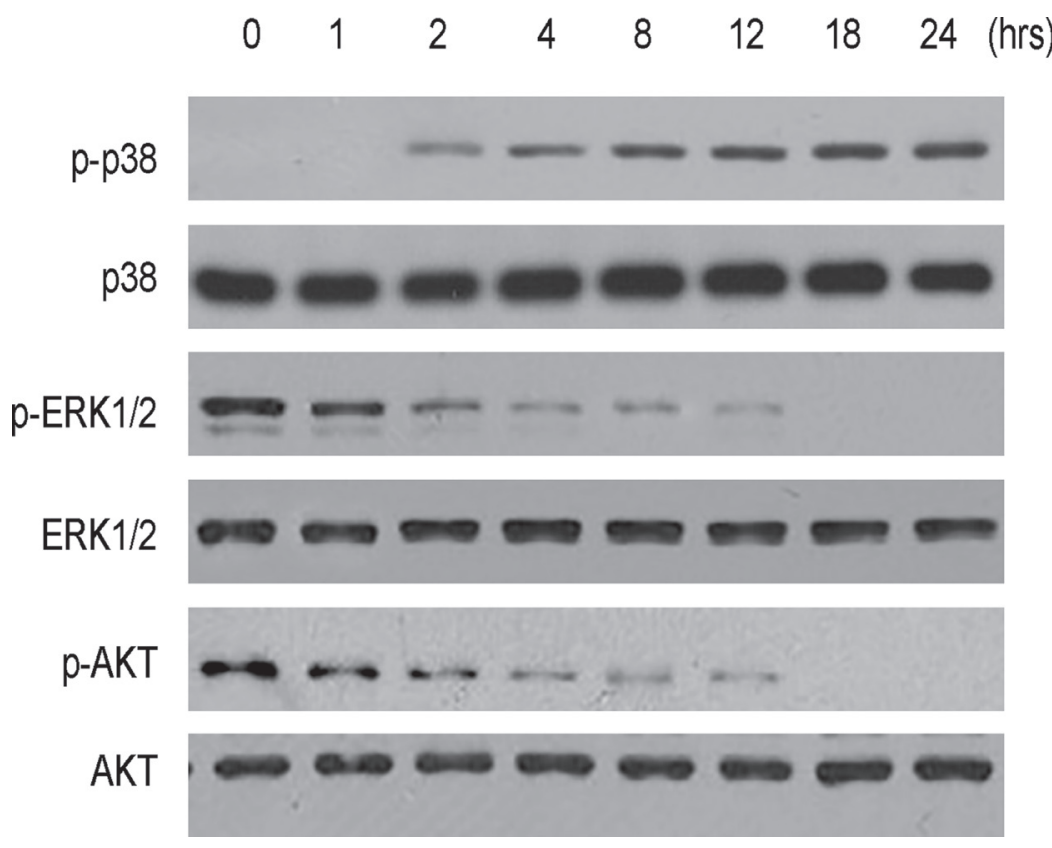

Figure 4: NDV-D90 enhances $\mathrm{p38}$ signaling and inhibits ERK1/2 and Akt signaling in gastric cancer cells. Representative Western blotting for phosphorylation of p38 (p-p38), p38, phosphorylation of ERK1/2 (p-ERK1/2), ERK1/2, phosphorylation of AKT and AKT after NDV-D90 treatment on BGC-823 cells. $N=5$. 
of NDV-D90 on tumor growth occurred earlier after treatment. In addition, the formation of the implanted tumor appeared to be affected by NDV-D90 treatment.

Although the current study investigated the effects of NDV-D90 on human gastric cancer cells, the tumor environment was in mice, but not in humans. The immunodeficiency state of nude mice may also have an effect on the interpretation of the data. Future studies may be performed in human patients to conquer these limitations. However, based on the collected evidence here, our data suggest that NDV-D90 may have potential anti-cancer effects on gastric cancer.

\section{MATERIALS AND METHODS}

\section{Protocol approval}

All the experimental methods in the current study has been approved by the research committee at Harbin Medical University. All the experiments have been carried out in accordance with the guidelines from the research committee at Harbin Medical University. All animal experiments were approved by the Institutional Animal Care and Use Committee at Harbin Medical University. Surgeries were performed in accordance with the
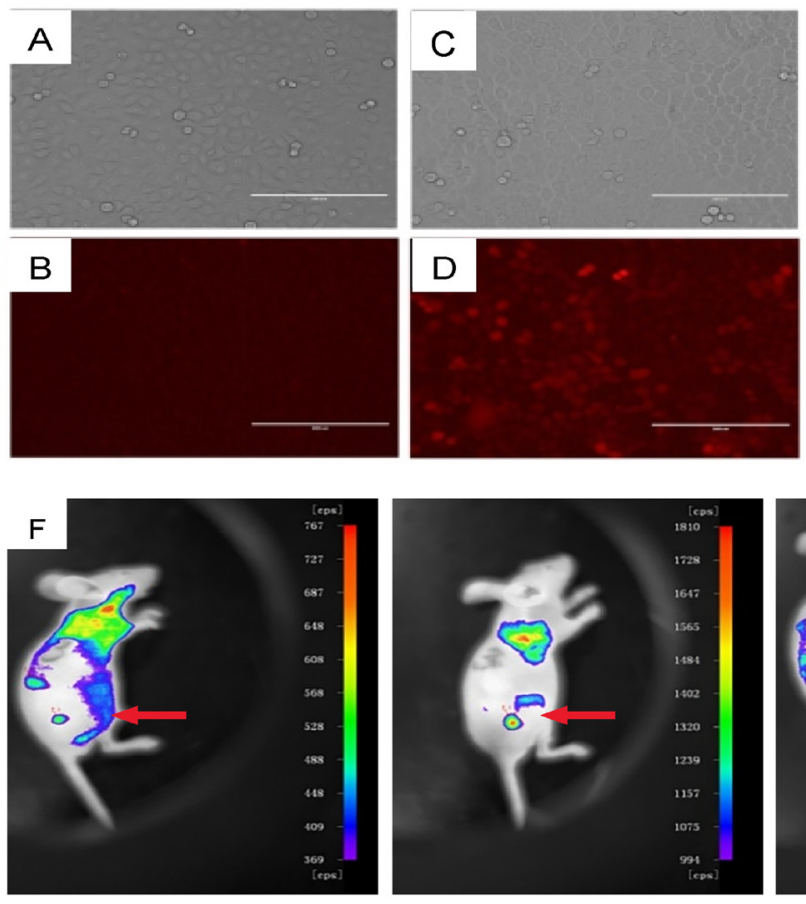

bvi

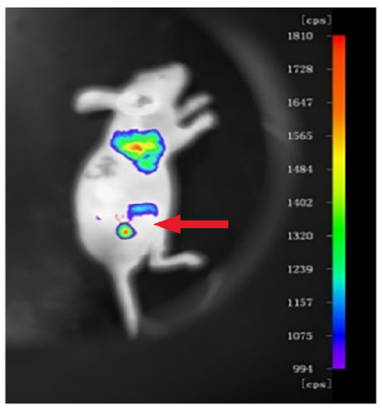

5 days pvi

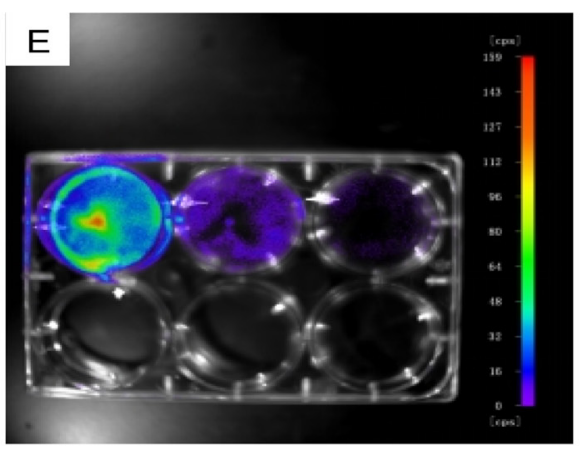

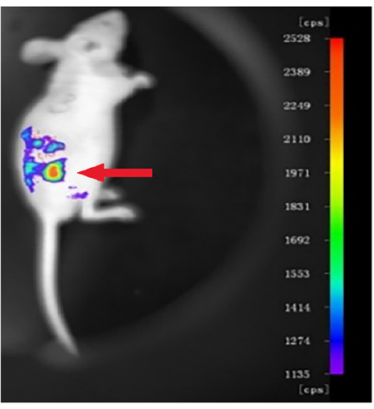

9 days pvi

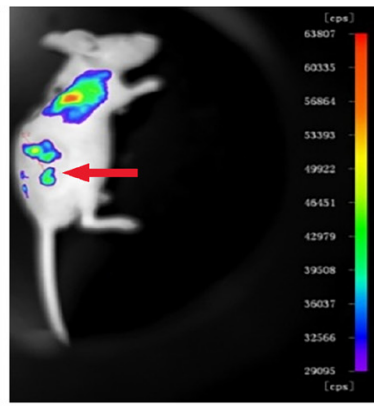

15 days pvi

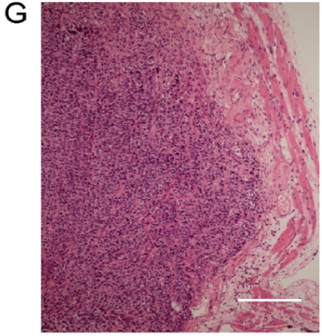

$\mathrm{OH}$

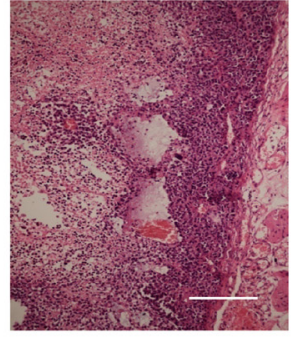

$48 \mathrm{H}$

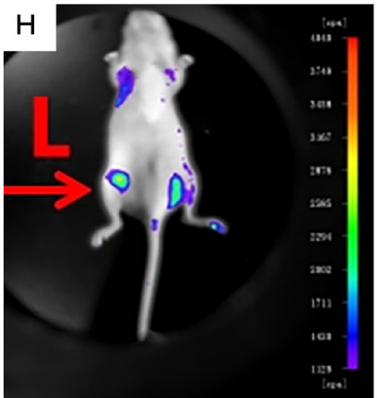

$\mathrm{OH}$

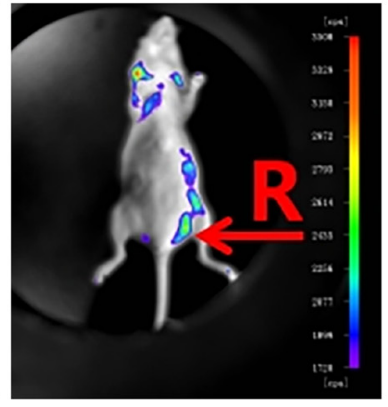

$48 \mathrm{H}$

Figure 5: NDV-D90 reduces gastric cancer cell growth in vivo. BGC-823 cells were transduced with lentivirus carrying a RFP reporter. (A) Bright field for non-transduced cells. (B) Red fluorescent field for non-transduced cells. (C) Bright field for transduced cells. (D) Red fluorescent field for transduced cells. (E) The transduced cells (termed as BGC-823-RFP) were validated under fluorescent microscopy in vitro. (F) BGC-823-RFP cells were then implanted into nude mice, after which NDV-D90 was intratumorally injected. The virally injected tumor (arrow) was monitored at different time points after viral injection, showing suppression of tumor growth by representative images. (G) Representative histological images showing presence of necrosis exclusively in the implanted BGC-823-RFP tumor treated with NDV-D90. (H) When BGC-823-RFP cells were pre-treated with NDV-D90 before implantation, the signals from the implanted tumor cells L: were hardly detected 48 hours after transplantation. R is control (BGC-823-RFP cells without NDV-D90 treatment). Bvi: before viral injection. Pvi: post viral injection. Scale bars are $100 \mu \mathrm{m} . N=5$. 
Principles of Laboratory Care, supervised by a qualified veterinarian.

\section{Reagents and cell line culture}

NDV-D90 was obtained from National Key Laboratory of Veterinary Biotechnology of Harbin Veterinary Medicine. Gastric cancer lines BGC-823, SGC7901 and MKN-28 were purchased from Chinese Academy of Sciences Culture Collection (Shanghai, China), and maintained in RPMI 1640 medium (Hyclone, Shanghai, China) supplemented with $10 \%$ fetal bovine serum (Hyclone) in a humidified chamber with $5 \% \mathrm{CO}_{2}$ at $37^{\circ} \mathrm{C}$.

\section{Cell viability assay}

The CCK-8 detection kit (DOJINDO, Shanghai, China) was used to measure cell viability. Briefly, gastric cancer cells were seeded in a 96-well microplate at a density of $10^{4} / \mathrm{ml}$. After successful attachment, cells were treated with NDV-D90 at a multiplicities of infection (MOI) of 0.001, 0.01, 0.1 and 1, respectively, for a duration of 2 hours. The cells were cultured for 12 hours, 24 hours, 36 hours, 48 hours, 60 hours and 72 hours, after which
$10 \mu \mathrm{l}$ CCK-8 solution was added in each well and the plate was incubated at $37^{\circ} \mathrm{C}$ for 4 hours before absorbance was measured with a monochromator microplate reader at a wavelength of $450 \mathrm{~nm}$. The optical density value was reported as the percentage of cell viability in relation to the control group (set as $100 \%$ ).

\section{Measurement of virus amplification}

Gastric cancer cells were seeded in a 6-well microplate at a density of $10^{5} / \mathrm{ml}$. After successful attachment, cells were treated with NDV-D90 at a MOI of 0.01 for a duration of 2 hours. The cells were cultured for 12 hours, 24 hours, 36 hours, 48 hours, 60 hours and 72 hours, and the culture supernatant was collected at each time point for measurement of the TCID . $_{50}$

\section{Tumor cell invasion test}

Gastric cancer cells were seeded in a 6-well microplate at a density of $10^{5} / \mathrm{ml}$. After successful attachment, cells were treated with NDV-D90 at a MOI of 0.01 for a duration of 2 hours. The infected cells were collected and $500 \mu \mathrm{l}$ was added into the upper chamber of
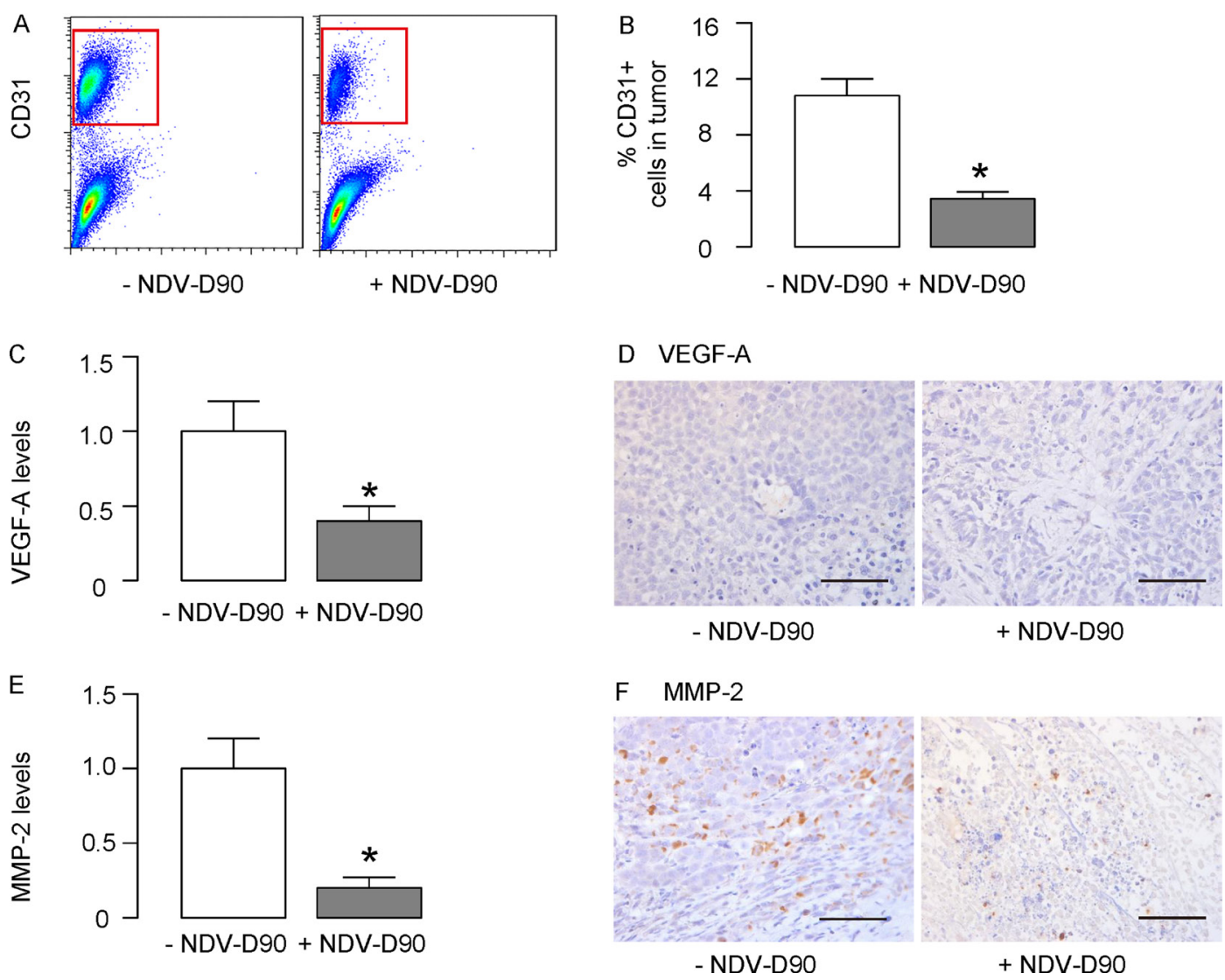

Figure 6: NDV-D90 impairs gastric cancer vascularization. (A-B) Implanted tumors treated with/without NDV-D90 were dissociated and analyzed for the percentage of CD31+ endothelial cells inside the tumor, shown by representative flow charts (A), and by quantification (B). (C-D) ELISA (C) and immunohistochemistry (D) for VEGF-A in implanted tumor. (E-F) ELISA (E) and immunohistochemistry (F) for MMP-2 in implanted tumor. 
a BD BioCoat ${ }^{\mathrm{TM}}$ Matrigel $^{\mathrm{TM}}$ Invasion Chamber (BectonDickinson Biosciences, San Jose, CA, USA) at a density of $5 \times 10^{4} / \mathrm{ml}$. The lower chamber was added with $750 \mu 1$ culture medium containing 10\% FBS. The incubation time was 14 hours, after which $50 \mu$ l Calcein-AM staining solution was added for 30 minutes to allow cells to be stained and visualized by fluorescent microscopy.

\section{Apoptosis assay and flow cytometry}

Cells were labeled with annexin V-FITC and propidium iodide (PI), using an apoptosis detecting kit (KeyGEN Biotech, Nanjing, China), and analyzed by flow cytometry using CellQuest software (Becton-Dickinson Biosciences, San Jose, CA, USA). For analyzing CD31+ cells in implanted tumor, the tumor was resected, minced into small pieces, and then digested in the digestion media containing $40 \mathrm{mg} / \mathrm{dl}$ collagenase (Sigma-aldrich, San Jose, CA, USA) and $0.05 \%$ trypsine (Sigma-Aldrich) at $37^{\circ} \mathrm{C}$ for $30 \mathrm{~min}$. After the digestion, the cells that passed a $40 \mu \mathrm{m}$ filter were subjected to flow cytometric analysis and sorting, using a FITC-conjugated rat-anti-mouse CD31 antibodiy (Becton-Dickinson Biosciences). Data were analyzed using FlowJo software (Flowjo LLC, Ashland, OR, USA).

\section{Western blot}

Protein was extracted from the cultured cells with RIPA lysis buffer (1\% NP40, 0.1\% Sodium dodecyl sulfate (SDS), $100 \mu \mathrm{g} / \mathrm{ml}$ phenylmethylsulfonyl fluoride, $0.5 \%$ sodium deoxycholate, in PBS) on ice. Protein concentration was determined using a BCA protein assay kit (Bio-rad, China), and whole lysates mixed with $4 \times$ SDS loading buffer (Bio-rad, China) were denatured by 5 minutes' incubation at $100^{\circ} \mathrm{C}$ for $5 \mathrm{~min}$. Afterwards, proteins were separated on SDS-polyacrylamide gels, and then transferred to a PVDF membrane. The membrane blots were first probed with a primary antibody. After incubation with horseradish peroxidase-conjugated second antibody, protein was visualized using an enhanced chemiluminescent system. Primary antibodies were rabbit anti-phosphorylated p38 (pp38), anti-p38, anti-phosphorylated ERK1/2 (p-ERK1/2), anti-ERK1/2, anti-phosphorylated AKT (p-AKT) and antiAKT (all purchased from Cell Signaling, San Jose, CA, USA). Secondary antibody is HRP-conjugated anti-rabbit (Jackson ImmunoResearch Labs, West Grove, PA, USA).

\section{ELISA}

ELISA was performed using mouse VEGF-A or MMP-2 ELISA kit (R\&D System, Los Angeles, CA, USA) according to manufacturer's instruction.

\section{Immunohistochemistry}

Immunohistochemistry was performed using a HRP/ DAB (ABC) Detection IHC kit (Abcam, Cambridge,
MA, USA) according to manufacturer's instruction. Both rabbit anti-VEGF-A and anti-MMP2 were purchased from R\&D System.

\section{Nude mouse tumor model}

BGC-823 cells were transduced with lenti-RFP viruses (ABM, Shanghai, China) to allow visualization of them in the implanted tumor by fluorescent microscopy. The male nude mice were purchased from SLAC Laboratory Animal Co. Ltd (Shanghai, China). These mice were used at 12 weeks of age, when they received subcutaneous transplantation with 108 gastric cancer cells on the back. After 3 weeks, the formed tumor received intratumoral injection of NDV-D90 or PBS as a control.

\section{Statistical analysis}

All statistical analyses were carried out using the SPSS 18.0 statistical software package. All data were statistically analyzed using one-way ANOVA with a Bonferroni correction, followed by Fisher's exact test to compare two groups. All values in cell and animal studies are depicted as mean \pm standard deviation and are considered significant if $p<0.05$.

\section{ACKNOWLEDGMENTS}

We thank to group of Dr. Xi Li from Harbin Veterinary Research Institute (HVRI) subordinated to the Chinese Academy of Agricultural Sciences (CAAS) for their generous provision of NDV-D90 and kind help of the experiments.

\section{CONFLICTS OF INTEREST}

The authors have declared that no competing interests exist.

\section{FUNDING}

This work was supported by National Natural Science Foundation of China (NO: 81201876), Heilongjiang Province Science Funds for Young Scholar (NO: QC2012C011), Heilongjiang Province Postdoctoral Science Foundation (NO: LBH-Z12210), Heilongjiang Provincial Health Bureau Projects (NO: 688) and Wu Jieping Medical Foundation Award (NO: 320.6750.15255).

\section{REFERENCES}

1. Pecqueux M, Fritzmann J, Adamu M, Thorlund $\mathrm{K}$, Kahlert C, Reissfelder C, Weitz J, Rahbari NN. Free intraperitoneal tumor cells and outcome in gastric cancer patients: a systematic review and meta-analysis. Oncotarget. 2015; 6:35564-35578. doi: 10.18632/oncotarget.5595. 
2. Riquelme I, Saavedra K, Espinoza JA, Weber H, Garcia P, Nervi B, Garrido M, Corvalan AH, Roa JC, Bizama C. Molecular classification of gastric cancer: Towards a pathway-driven targeted therapy. Oncotarget. 2015; 6:24750-24779. doi: 10.18632/oncotarget.4990.

3. Matumoto M. Enhanced replication of Newcastle disease virus in cell culture co-infected with certain other viruses. Jpn J Microbiol. 1968; 12:505-530.

4. Tan L, Zhang Y, Zhan Y, Yuan Y, Sun Y, Qiu X, Meng C, Song C, Liao Y, Ding C. Newcastle disease virus employs macropinocytosis and Rab5a-dependent intracellular trafficking to infect DF-1 cells. Oncotarget. 2016; 7:86117-86133. doi: 10.18632/oncotarget.13345.

5. Vigil A, Park MS, Martinez O, Chua MA, Xiao S, Cros JF, Martinez-Sobrido L, Woo SL, Garcia-Sastre A. Use of reverse genetics to enhance the oncolytic properties of Newcastle disease virus. Cancer Res. 2007; 67:8285-8292.

6. Csatary LK, Gosztonyi G, Szeberenyi J, Fabian Z, Liszka V, Bodey B, Csatary CM. MTH-68/H oncolytic viral treatment in human high-grade gliomas. J Neurooncol. 2004; 67:83-93.

7. Freeman AI, Zakay-Rones Z, Gomori JM, Linetsky E, Rasooly L, Greenbaum E, Rozenman-Yair S, Panet A, Libson E, Irving CS, Galun E, Siegal T. Phase I/II trial of intravenous NDV-HUJ oncolytic virus in recurrent glioblastoma multiforme. Mol Ther. 2006; 13:221-228.

8. Lorence RM, Roberts MS, O’Neil JD, Groene WS, Miller JA, Mueller SN, Bamat MK. Phase 1 clinical experience using intravenous administration of PV701, an oncolytic Newcastle disease virus. Curr Cancer Drug Targets. 2007; 7:157-167.

9. Laurie SA, Bell JC, Atkins HL, Roach J, Bamat MK, O’Neil JD, Roberts MS, Groene WS, Lorence RM. A phase 1 clinical study of intravenous administration of PV701, an oncolytic virus, using two-step desensitization. Clin Cancer Res. 2006; 12:2555-2562.

10. Prince HM, Regester G, Gates P, Jablonskis L, Seymour JF, Lillie K, West R, Wolf M, Januszewicz H, Belford D. A phase Ib clinical trial of PV701, a milk-derived protein extract, for the prevention and treatment of oral mucositis in patients undergoing high-dose BEAM chemotherapy. Biol Blood Marrow Transplant. 2005; 11:512-520.

11. Pecora AL, Rizvi N, Cohen GI, Meropol NJ, Sterman D, Marshall JL, Goldberg S, Gross P, O’Neil JD, Groene WS, Roberts MS, Rabin H, Bamat MK, et al. Phase I trial of intravenous administration of PV701, an oncolytic virus, in patients with advanced solid cancers. J Clin Oncol. 2002; 20:2251-2266.

12. Fu F, Zhao M, Yang YJ, Tong GZ, Yang BF, Song C, Li X. Antiproliferative effect of newcastle disease virus strain D90 on human lung cancer cell line A549. Oncol Res. 2011; 19:323-333.

13. Chai Z, Zhang P, Fu F, Zhang X, Liu Y, Hu L, Li X. Oncolytic therapy of a recombinant Newcastle disease virus D90 strain for lung cancer. Virology journal. 2014; 11:84.

14. Zhang CX, Ye LW, Liu Y, Xu XY, Li DR, Yang YQ, Sun LL, Yuan J. Antineoplastic activity of Newcastle disease virus strain D90 in oral squamous cell carcinoma. Tumour Biol. 2015; 36:7121-7131.
15. Barnes BJ, Moore PA, Pitha PM. Virus-specific activation of a novel interferon regulatory factor, IRF-5, results in the induction of distinct interferon alpha genes. J Biol Chem. 2001; 276:23382-23390.

16. Gao M, Zhang X, Li D, He P, Tian W, Zeng B. Expression analysis and clinical significance of eIF4E, VEGF-C, E-cadherin and MMP-2 in colorectal adenocarcinoma. Oncotarget. 2016; 7:85502-85514. doi: 10.18632/ oncotarget.13453.

17. Chen YS, Meng F, Li HL, Liu QH, Hou PF, Bai J, Zheng JN. Dicer suppresses MMP-2-mediated invasion and VEGFA-induced angiogenesis and serves as a promising prognostic biomarker in human clear cell renal cell carcinoma. Oncotarget. 2016; 7:84299-84313. doi: 10.18632/oncotarget.12520.

18. Li HL, Han L, Chen HR, Meng F, Liu QH, Pan ZQ, Bai J, Zheng JN. PinX1 serves as a potential prognostic indicator for clear cell renal cell carcinoma and inhibits its invasion and metastasis by suppressing MMP-2 via NF-kappaBdependent transcription. Oncotarget. 2015; 6:21406-21420. doi: 10.18632/oncotarget.4011.

19. Wang Z, Cao CJ, Huang LL, Ke ZF, Luo CJ, Lin ZW, Wang F, Zhang YQ, Wang LT. EFEMP1 promotes the migration and invasion of osteosarcoma via MMP-2 with induction by AEG-1 via NF-kappaB signaling pathway. Oncotarget. 2015; 6:14191-14208. doi: 10.18632/ oncotarget.3691.

20. Gonzalez-Villasana V, Fuentes-Mattei E, Ivan C, Dalton HJ, Rodriguez-Aguayo C, Fernandez-de Thomas RJ, Aslan B, Del CMP, Velazquez-Torres G, Previs RA, Pradeep S, Kahraman N, Wang H, et al. Rac1/Pak1/p38/MMP-2 Axis Regulates Angiogenesis in Ovarian Cancer. Clin Cancer Res. 2015; 21:2127-2137.

21. Kim BS, Park JY, Kang HJ, Kim HJ, Lee J. Fucoidan/FGF-2 induces angiogenesis through $\mathrm{JNK}$ - and p38-mediated activation of AKT/MMP-2 signalling. Biochem Biophys Res Commun. 2014; 450:1333-1338.

22. Lu W, Xia YH, Qu JJ, He YY, Li BL, Lu C, Luo X, Wan XP. p21-activated kinase 4 regulation of endometrial cancer cell migration and invasion involves the ERK1/2 pathway mediated MMP-2 secretion. Neoplasma. 2013; 60:493-503.

23. Zhang Z, Zhang H, Peng T, Li D, Xu J. Melittin suppresses cathepsin $\mathrm{S}$-induced invasion and angiogenesis via blocking of the VEGF-A/VEGFR-2/MEK1/ERK1/2 pathway in human hepatocellular carcinoma. Oncology letters. 2016; 11:610-618.

24. Walczak C, Gaignier F, Gilet A, Zou F, Thornton SN, Ropars A. Aldosterone increases VEGF-A production in human neutrophils through PI3K, ERK1/2 and p38 pathways. Biochim Biophys Acta. 2011; 1813:2125-2132.

25. Lee HP, Lin CY, Shih JS, Fong YC, Wang SW, Li TM, Tang CH. Adiponectin promotes VEGF-A-dependent angiogenesis in human chondrosarcoma through PI3K, Akt, mTOR, and HIF-alpha pathway. Oncotarget. 2015; 6:36746-36761. doi: 10.18632/oncotarget.5479. 